\title{
The Surface EMG Characteristics between Erector Spinae and Vastus Lateralis during Bending Forward and Squatting Down Tasks
}

\author{
Jinzhuang Xiao, ${ }^{1}$ Junmin Gao, ${ }^{1}$ Hongrui Wang, ${ }^{1}$ Kun Liu, ${ }^{1}$ and Xincai Yang ${ }^{2}$ \\ ${ }^{1}$ College of Electronic and Information Engineering, Hebei University, Baoding, Hebei 071000, China \\ ${ }^{2}$ Healthy Rehabilitation Section, Affiliated Hospital of Hebei University, Baoding, Hebei 071000, China \\ Correspondence should be addressed to Jinzhuang Xiao; robot@hbu.edu.cn
}

Received 26 May 2015; Revised 21 September 2015; Accepted 5 October 2015

Academic Editor: Richard H. Bayford

Copyright (C) 2015 Jinzhuang Xiao et al. This is an open access article distributed under the Creative Commons Attribution License, which permits unrestricted use, distribution, and reproduction in any medium, provided the original work is properly cited.

Bending forward and squatting down are two common physical tasks in everyday life and at work. This study determined the sEMG characteristics between erector spinae and vastus lateralis during bending forward and squatting down tasks by analyzing the regularity of variation in sEMG frequency domain index-median frequency (MNF) in healthy subjects. The results indicate that sEMG activities in erector spinae are significantly higher than those in the right vastus lateralis during bending forward task. Instead, in squatting down task, sEMG activities in the right vastus lateralis are significantly greater than those in erector spinae. It also shows that the fatigue degree of low back caused by trunk bending is much deeper than that by squatting down. The ratio scope of one-order fitting values of MNF between erector spinae and vastus lateralis ranges from 0.87 to 1.72 among $80 \%$ of subjects during bending forward task and ranges from 0.31 to 1.11 during squatting down task. This study provides certain theoretical basis for using the strength of low back in a proper way to avoid getting it hurt in daily physical activities.

\section{Introduction}

Bending forward is a common physical task in everyday life and at work. For example, people must bend down when they carry heavy loads or pick up something on the ground. When people do the housework, they often keep their bodies in forward flexion condition. In these cases, if people do not pay attention to their posture, especially for those who lack exercise, it is easy to cause damage to low back. What is more, using the strength of low back incorrectly and prolonged trunk bending have been treated as risk factors for low back pain (LBP). Due to increased pain and/or muscle weakness, individuals with LBP often have difficulty in performing trunk bending and lifting task. Low back pain (LBP) is one of the most general public health problems in today's society and makes a considerable contribution to disability [1-3]. Hence, it is extremely essential for people to adjust their posture in a proper way. In order to avoid aggravating the burden of low back, people with or without LBP will consciously rely on the power of lower limbs muscle to implement some actions, such as squatting down.

Surface electromyography (sEMG) has been used in both research and clinical applications for noninvasive neuromuscular assessment in several different fields such as sport science, neurophysiology, and rehabilitation [4-6]. The use of surface electromyography (sEMG) techniques has played a major role in understanding of the functional activity of lumbar muscles in both healthy and LBP subjects [7]. SEMG has been widely utilized to study changes in trunk and lower extremity muscle activities [8-10].

As one of the essential parameters for the analysis of electromyography signals, frequency domain analysis was used in this study. In this research, fast Fourier transform (FFT) and classical power spectra were used to process the filtered sEMG signals and obtain the features of power spectra. Median frequency (MNF) and median frequency slope (MNF SLO) are usually used as quantifications of the power spectrum [11-13]. Mean frequency is the average frequency 
value of the power spectrum, while median frequency (MNF) is defined as the frequency that separates the power spectrum into halves of equal power $[14,15]$. Median frequency slope (MNF SLO) reflects fatigability during prolonged exercise [16].

A few of previous studies have shown the evaluation of sEMG characteristics of low back and lower limbs between the patients with LBP and healthy subjects. A recent study showed that the normalized EMG activity in erector spinae and the right gluteus maximus was significantly higher in the LBP group than in the healthy group during prone hip extension [9]. Farahpour et al. analyzed the EMG responses of erector spinae and lower limbs muscles to unexpected forward and backward perturbation test in patients with adolescent idiopathic scoliosis and healthy volunteers. The results presented that, in forward postural perturbation test, the EMG responses of erector spinae and biceps femoris muscles in the adolescent idiopathic scoliosis group were greater than those in control group [17]. Leinonen et al. compared lumbar paraspinal, gluteus maximus, and biceps femoris muscle function during sagittal trunk flexion and extension in patients with chronic low back pain and healthy control subjects. The results showed that activities of gluteus maximus muscle during the flexion-extension cycle were reduced in patients with chronic low back pain [18]. MasséAlarie et al. assessed the onset timing of the transverses abdominis/internal oblique muscles during two rapid limb movement tasks with different postural demands as well as differences between CLBP and controls. In both groups, 50\% of bilateral shoulder flexion trials showed bilateral anticipatory $\operatorname{Tr} \mathrm{A} / \mathrm{IO}$ activation. But in unilateral hip extension only the $\operatorname{TrA} / \mathrm{IO}$ contralateral to the moving leg showed anticipatory activation [19]. However, these studies did not focus on the correlation in muscular activity between low back and lower limbs. Moreover, there are fewer data about the sEMG characteristics between low back and lower limbs in daily physical activities, such as bending forward and squatting down.

The objective of this study was to determine the sEMG characteristics between erector spinae and vastus lateralis during bending forward and squatting down tasks in healthy subjects by analyzing the regularity of variation in sEMG frequency domain index-median frequency (MNF). Due to the aim of this research which was to distinguish which one could lower the fatigue degree of low back muscle during two tasks, this study only chose the healthy individuals for the experiment. Moreover, according to the requirements, it is somewhat difficult for parts of LBP individuals to implement the experiment uniformly. The study provides certain theoretical basis for using the strength of low back in a proper way to avoid getting it hurt in daily physical activities.

\section{Methodology}

2.1. Subjects. Seven male and three female volunteers participated in this investigation. All subjects were healthy with normal physical characteristics (see Table 1) and had no history of pain in lower limbs or low back. None of them
TABLE 1: Subjects anthropometric characteristics.

\begin{tabular}{lccc}
\hline Gender & Age (year) & Height $(\mathrm{cm})$ & Mass $(\mathrm{kg})$ \\
\hline Male & $25.0 \pm 2.0$ & $175.0 \pm 2.6$ & $77.5 \pm 10.5$ \\
Female & $25.0 \pm 1.0$ & $165.0 \pm 3.2$ & $50.5 \pm 2.4$ \\
\hline
\end{tabular}

participated in any activities in the past 24 hours before the test. Prior to the study, all subjects received verbal and written information about the study. This study was approved by the Biomedical Multidisciplinary Research Center of affiliated university.

2.2. Procedure. The implementation of this study consisted of two exercise movement tasks. In the first task, the subjects were required to stand with feet shoulder width apart and drop their arms naturally at both sides with a pair of $1.25 \mathrm{~kg}$ dumbbells in hand. When the subject heard the order "start," the upper body bent forward about $90^{\circ}$ and then restored upright position, and so the subject repeated this action every 3 seconds for a minute. In the process of reciprocating, the arms must keep straight down, as shown in Figure 1(a).

In the second task, the subjects were asked to hold a pair of $1.25 \mathrm{~kg}$ dumbbells. When the subject heard the order "start," he or she put his or her left foot flat on the floor, right foot moved back half a step, the body squatted down naturally, shifted centre of gravity of the hip onto the right foot, and then restored upright position, and so he or she repeated this action every 3 seconds for a minute (see Figure 1(b)). Similarly, in the process of squatting down and standing up, the arms must keep straight down.

In this study, sEMG signals were recorded on erector spinae and vastus lateralis [20]. A pair of $\mathrm{Ag}-\mathrm{AgCl}$ electrodes were attached to the right erector spinae prominences which were at L2 L3 segment of the vertebra along the longitudinal axis of the erector spinae and the vastus lateralis on the right leg. Electrode diameter was $0.5 \mathrm{~cm}$ and electrode pitch was $2.0 \mathrm{~cm}$. The reference electrode was placed on the outside of the vertical between the two electrodes. Before attaching surface electrodes, electrodes sites were cleaned with alcohol and shaved with disposable shavers. After that, the skin was abraded by soft sand paper and then again cleaned with alcohol to ensure reducing skin impedance which is reduced from $3 \mathrm{M} \Omega$ to less than $500 \mathrm{k} \Omega$. Electromyography signals from subjects were collected using the Shimmer Development Kit which was provided by the Shimmer Corporation of Ireland, as shown in Figure 1(a). The Shimmer collected sEMG continuously for 60 seconds and transferred data into a personal computer via Bluetooth wireless transmission in real time. All data collected were stored as.csv file automatically. This file could be opened by Microsoft Excel, from which the stored sEMG data were decoded.

2.3. Data Analysis and Processing. The raw sEMG signals were recorded at a sampling rate of $1000 \mathrm{~Hz}$ and filtered by using a four-order Butterworth filter to produce a bandwidth of $10-500 \mathrm{~Hz}$. A two-order notch filter with a band stop of $49-51 \mathrm{~Hz}$ was used to eliminate AC line interference. 


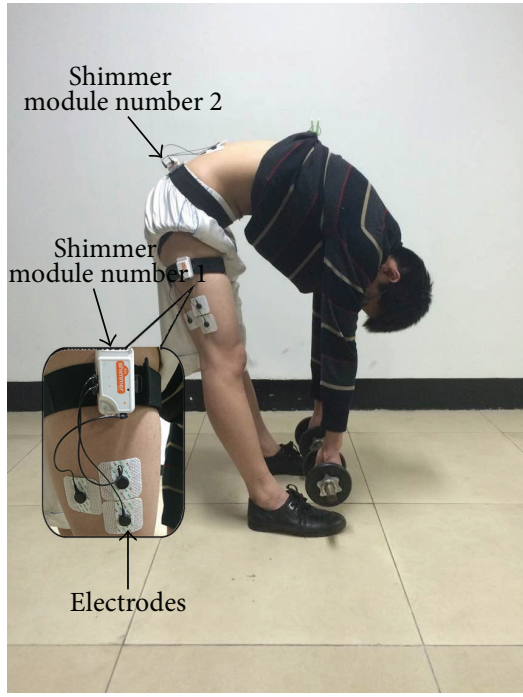

(a)

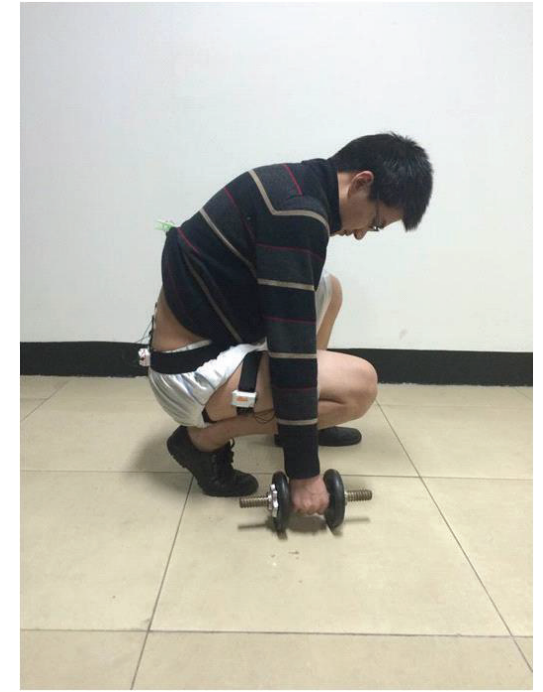

(b)

FIGURE 1: (a) Bending forward. (b) Squatting down.

The recorded sEMG signals that were stored in the computer were divided by intervals of $3 \mathrm{~s}$. Median frequency (MNF) of the sEMG power spectrum was calculated at each $3 \mathrm{~s}$ interval with fast Fourier transform (FFT) algorithms.

SEMG signals were used to calculate MNF by the following equation:

$$
\begin{aligned}
\mathrm{MNF} & =\frac{1}{2} \int_{0}^{\infty} S(f) d f=\int_{0}^{\mathrm{MNF}} S(f) d f \\
& =\int_{\mathrm{MNF}}^{\infty} S(f) d f,
\end{aligned}
$$

where MNF means median frequency, $f$ is the frequency, $S(f)$ is the power spectrum, $d f$ is the frequency resolution.

The processing of sEMG and the calculation of median frequency (MNF) were achieved in MATLAB R2009b.

\section{Results}

The statistical results indicated that about $80 \%$ of subjects' muscle activities showed consistency. It showed that there was an increase in the amplitude of lower frequency band and a relative decrease in higher frequency band in the power spectrum of sEMG signals. In Figures 2 and 3, the horizontal axis represented time, which was divided into 20 segments with $3 \mathrm{~s}$ per segment, and the vertical axis represented the MNF value of each segment. The average of MNF of $80 \%$ subjects was shown using curve.

Figure 2 presented the respective change of median frequency (MNF) and its one-order fitting values of erector spinae and vastus lateralis in the first task during 60 seconds. The values of MNF showed a decline trend in a fluctuating way with times going among erector spinae and vastus lateralis. But the absolute value of one-order fitting in erector spinae was greater than that in vastus lateralis.

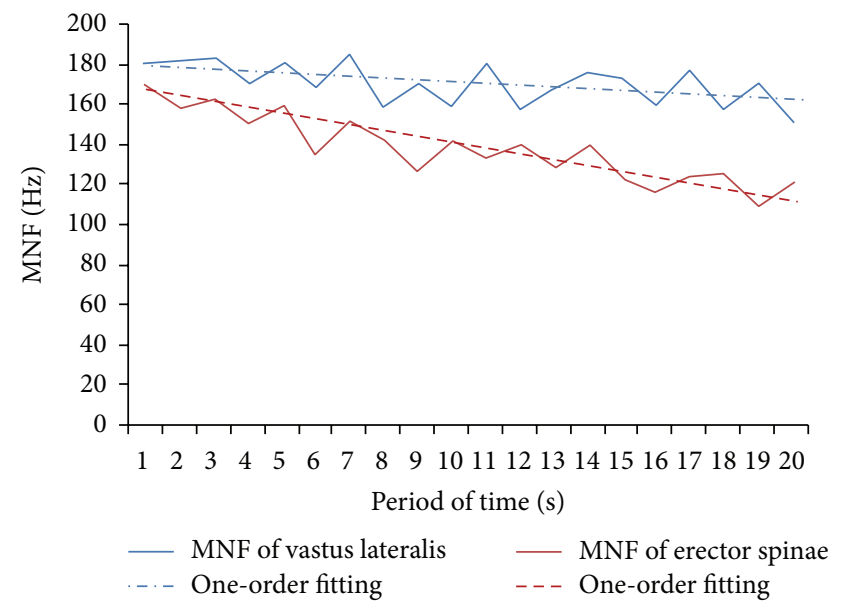

Figure 2: The MNF and its one-order fitting of erector spinae and vastus lateralis in the first task.

Figure 3 showed the respective change of MNF and its one-order fitting values of erector spinae and vastus lateralis in the second task. Different from Figure 2, the absolute value of one-order fitting in vastus lateralis was greater than that in erector spinae.

Figure 4 showed the ratio of one-order fitting values of MNF between erector spinae and vastus lateralis among $80 \%$ of subjects in two tasks. The bar chart revealed that the ratio scope ranged from 0.87 to 1.72 in the first task and ranged from 0.31 to 1.11 in the second task.

\section{Discussion}

This study demonstrates the difference in the muscle activities between erector spinae and vastus lateralis while performing the given two tasks. Numerous studies have used sEMG to 


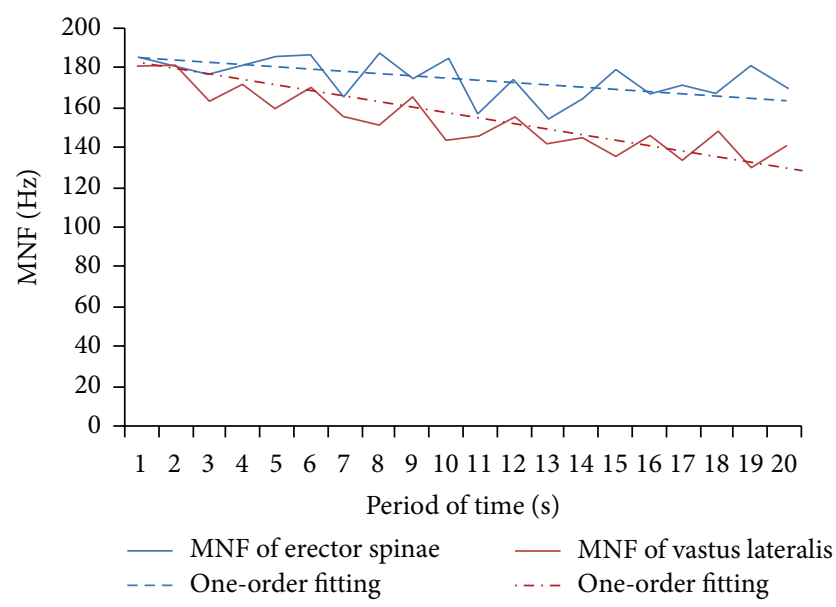

Figure 3: The MNF and its one-order fitting of erector spinae and vastus lateralis in the second task.

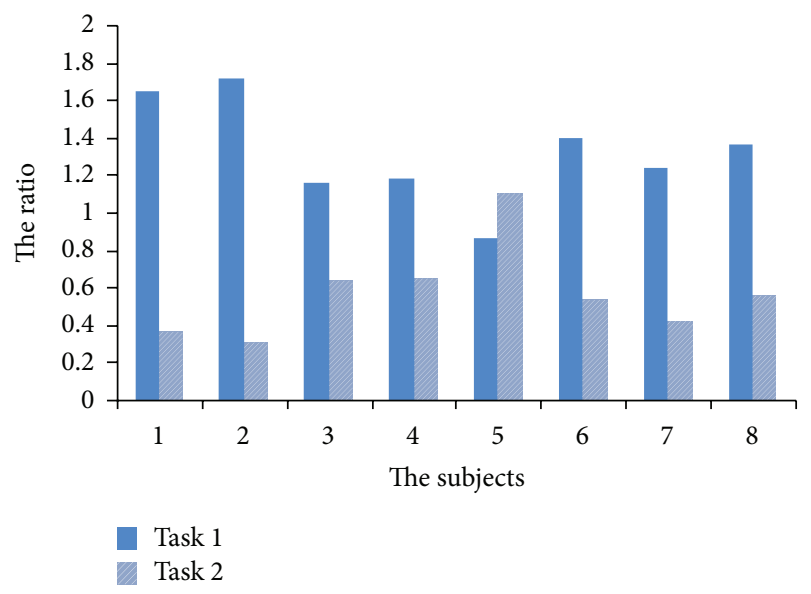

Figure 4: The ratio of one-order fitting values of MNF.

identify muscle fatigue. In a previous study, it is suggested that there is a concomitant change in the power spectrum of sEMG signals during muscle fatigues. The shift towards lower frequency in sEMG power spectrum has been found to be associated with localized muscle fatigue. Thus, it results in the decrease of MNF in the frequency domain. It has been reported that muscle fatigue can be determined based on the reduction of MNF. Median frequency (MNF) has been found to have advantages over other sEMG assessment techniques such as amplitude. And median frequency (MNF) is a reliable and accurate measure of the frequency shift of the sEMG in muscle fatigue [21-24]. This research also indicates that there is an increase in the amplitude of the lower frequency band and a relative decrease in the higher frequency band in the power spectrum of sEMG signals. Figures 2 and 3 present the decline of MNF with the subjects feeling muscle fatigue over time. These results keep consistency with previous conclusions.

In a previous study, it shows that although central and peripheral factors are responsible, the main factor contributing to the MNF shift towards lower values may be peripheral, that is, a slowing of the muscle action potential conduction velocity [25].

It is evident from Figure 2 that the decline of median frequency (MNF) in erector spinae is much faster than the decline of median frequency (MNF) in vastus lateralis. The result demonstrates that sEMG activities in erector spinae are significantly higher than those in the right vastus lateralis. It further illuminates that subjects easily feel more fatigue in low back than in lower limbs during bending forward task. Instead, the decline of median frequency (MNF) in vastus lateralis is much faster than the decline of median frequency (MNF) in erector spinae in Figure 3. Similarly, subjects easily feel more fatigue in lower limbs than in low back during squatting down task.

In the bending forward, the burden of low back is more heavier than that of lower limbs. However, in squatting down task, the burden of lower limbs becomes even heavier. Figures 2 and 3 show that the fatigue degree of low back caused by trunk bending is much deeper than that caused by squatting down. Hence, people are supposed to replace trunk bending with squatting down appropriately to avoid aggravating the burden of low back.

Figure 4 presents the ratio of one-order fitting values of MNF between erector spinae and vastus lateralis among $80 \%$ of subjects in two tasks. The bar chart which represents the ratio values between erector spinae and vastus lateralis reveals that ratio scope ranges from 0.87 to 1.72 in the first task and ranges from 0.31 to 1.11 in the second task. These results suggest that there are certain correlations between erector spinae and vastus lateralis in the muscle activities.

In the literature, most authors find no statistically significant difference in muscle fiber compositions between genders [26]. In this study, male and female subjects show consistency with the regularity of variation of median frequency (MNF).

The deficiency of this study is that subjective factors have some influences on the experimental results and, due to taking heavy lumbar load, it is somewhat difficult for the parts of LBP individuals to implement the bending forward task. Therefore, the application scope of this experiment is limited. How to improve the experiment is to be further investigated.

\section{Conclusions}

This study determined the sEMG characteristics between erector spinae and vastus lateralis during bending forward and squatting down tasks in healthy subjects by analyzing the regularity of variation in sEMG frequency domain indexmedian frequency (MNF). It showed that $80 \%$ of subjects' muscle activities showed consistency. During bending forward task, sEMG activities in erector spinae are significantly higher than those in the right vastus lateralis. Subjects easily feel more fatigue in low back than in lower limbs. Instead, in squatting down task, sEMG activities in the right vastus lateralis are significantly greater than those in erector spinae. Subjects easily feel more fatigue in lower limbs than in low back. The ratio scope between low back and lower limbs ranges from 0.87 to 1.72 in the first task and ranges from 0.31 to 1.11 in the second task. 
The application scope of this experiment is limited due to the influence of subjective factors and it is somewhat difficult for parts of LBP individuals to implement the experiment.

In daily life, people are supposed to replace trunk bending with squatting down appropriately to avoid low back pain (LBP). This study provides certain theoretical basis for using the strength of low back in a proper way to avoid getting it hurt in everyday physical activities.

\section{Conflict of Interests}

The authors declare that there is no conflict of interests regarding the publication of this paper.

\section{Acknowledgments}

The authors wish to thank all the members of Biomedical Multidisciplinary Research Center and the volunteers who participated in this study and the grant of Key Laboratory of Digital Medical Engineering of Hebei Province, China. Thanks also are due to Doctor Zhao, Baoding Rehabilitation Center, Hebei Province, China, for the support and direction.

\section{References}

[1] F. M. Feldwieser, L. Sheeran, A. Meana-Esteban, and V. Sparkes, "Electromyographic analysis of trunk-muscle activity during stable, unstable and unilateral bridging exercises in healthy individuals," European Spine Journal, vol. 21, no. 2, pp. 171-186, 2012.

[2] N. R. Marques, M. H. Morcelli, C. Z. Hallal, and M. Gonçalves, "EMG activity of trunk stabilizer muscles during centering principle of pilates method," Journal of Bodywork and Movement Therapies, vol. 17, no. 2, pp. 185-191, 2013.

[3] R. J. Butler and W. G. Johnson, "Adjusting rehabilitation costs and benefits for health capital: the case of low back occupational injuries," Journal of Occupational Rehabilitation, vol. 20, no. 1, pp. 90-103, 2010.

[4] S. Donaldson, M. Donaldson, and L. Snelling, "SEMG evaluations: an overview," Applied Psychophysiology and Biofeedback, vol. 28, no. 2, pp. 121-127, 2003.

[5] B. Van Damme, V. Stevens, D. Van Tiggelen, C. Perneel, G. Crombez, and L. Danneels, "Performance based on sEMG activity is related to psychosocial components: differences between back and abdominal endurance tests," Journal of Electromyography and Kinesiology, vol. 24, no. 5, pp. 636-644, 2014.

[6] R. Grassme, D. Arnold, Ch. Anders et al., "Improved evaluation of back muscle SEMG characteristics by modelling," Pathophysiology, vol. 12, no. 4, pp. 307-312, 2005.

[7] H. Zabaleta, C. Rodriguez-de-Pablo, N. Miljkovic, T. Keller, and G. A. Garcia, "sEMG-based detection of poor posture: a feasibility study," in Proceedings of the 34th Annual International Conference of the IEEE Engineering in Medicine and Biology Society (EMBS '12), pp. 1210-1213, IEEE, San Diego, Calif, USA, September 2012.

[8] Y. S. S. M. Al-Khabbaz, T. Shimada, and M. Hasegawa, "The effect of backpack heaviness on trunk-lower extremity muscle activities and trunk posture," Gait \& Posture, vol. 28, no. 2, pp. 297-302, 2008.
[9] J.-W. Kim, O.-Y. Kwon, T.-H. Kim, D.-H. An, and J.-S. Oh, "Effects of external pelvic compression on trunk and hip muscle EMG activity during prone hip extension in females with chronic low back pain," Manual Therapy, vol. 19, no. 5, pp. 467471, 2014.

[10] P. Heinonen, H. Kautiainen, and M. Mikkelsson, "Erector spinae SEMG activity during forward flexion and re-extension in ankylosing spondylitis patients," Pathophysiology, vol. 12, no. 4, pp. 289-293, 2005.

[11] C. Demoulin, J.-M. Crielaard, and M. Vanderthommen, "Spinal muscle evaluation in healthy individuals and low-back-pain patients: a literature review," Joint Bone Spine, vol. 74, no. 1, pp. 9-13, 2007.

[12] G. T. Allison and T. Fujiwara, "The relationship between sEMG median frequency and low frequency band amplitude changes at different levels of muscle capacity," Clinical Biomechanics, vol. 17, no. 6, pp. 464-469, 2002.

[13] I. R. T. de Jesus, R. G. T. Mello, and J. Nadal, "Principal components of frequency domain electromyograms for muscular fatigue analysis," in Proceedings of the IEEE 32nd Annual International Conference of the Engineering in Medicine and Biology Society (EMBC '10), pp. 3519-3522, Buenos Aires, August 2010.

[14] F. B. Stulen and C. J. Deluca, "Frequency parameters of the myoelectric signal as a measure of muscle conduction velocity," IEEE Transactions on Biomedical Engineering, vol. 28, no. 7, pp. 515-523, 1981.

[15] M. Cifrek, V. Medved, S. Tonković, and S. Ostojić, "Surface EMG based muscle fatigue evaluation in biomechanics," Clinical Biomechanics, vol. 24, no. 4, pp. 327-340, 2009.

[16] B. Elfving, D. Liljequist, Å. Dedering, and G. Németh, "Recovery of electromyograph median frequency after lumbar muscle fatigue analysed using an exponential time dependence model," European Journal of Applied Physiology, vol. 88, no. 1-2, pp. 8593, 2002.

[17] N. Farahpour, S. Ghasemi, P. Allard, and M. S. Saba, "Electromyographic responses of erector spinae and lower limb's muscles to dynamic postural perturbations in patients with adolescent idiopathic scoliosis," Journal of Electromyography and Kinesiology, vol. 24, no. 5, pp. 645-651, 2014.

[18] V. Leinonen, M. Kankaanpää, O. Airaksinen, and O. Hänninen, "Back and hip extensor activities during trunk flexion/extension: effects of low back pain and rehabilitation," Journal of Archives of Physical Medicine and Rehabilitation, vol. 81, no. 1, pp. 32-37, 2000.

[19] H. Massé-Alarie, L. Beaulieu, R. Preuss, and C. Schneider, "Task-specificity of bilateral anticipatory activation of the deep abdominal muscles in healthy and chronic low back pain populations," Gait \& Posture, vol. 41, no. 2, pp. 440-447, 2015.

[20] A. Rainoldi, G. Melchiorri, and I. Caruso, "A method for positioning electrodes during surface EMG recordings in lower limb muscles," Journal of Neuroscience Methods, vol. 134, no. 1, pp. 37-43, 2004.

[21] T. Tsuboi, T. Satou, K. Egawa, Y. Izumi, and M. Miyazaki, "Spectral analysis of electromyogram in lumbar muscles: fatigue induced endurance contraction," European Journal of Applied Physiology, vol. 69, no. 4, pp. 361-366, 1994.

[22] P. S. Sung, A. R. Lammers, and P. Danial, "Different parts of erector spinae muscle fatigability in subjects with and without low back pain," Spine Journal, vol. 9, no. 2, pp. 115-120, 2009.

[23] M. A. M. Bandpei, N. Rahmani, B. Majdoleslam, I. Abdollahi, S. S. Ali, and A. Ahmad, "Reliability of surface electromyography 
in the assessment of paraspinal muscle fatigue: an updated systematic review," Journal of Manipulative and Physiological Therapeutics, vol. 37, no. 7, pp. 510-521, 2014.

[24] M. Ali, M. Bandpei, and M. J. Watson, "Electromyographic power spectral analysis of the paraspinal muscles," Physiotherapy, vol. 87, no. 9, pp. 470-478, 2001.

[25] V. Balasubramanian and S. Jayaraman, "Surface EMG based muscle activity analysis for aerobic cyclist," Journal of Bodywork and Movement Therapies, vol. 13, no. 1, pp. 34-42, 2009.

[26] C. Anders, S. Bretschneider, A. Bernsdorf, K. Erler, and W. Schneider, "Activation of shoulder muscles in healthy men and women under isometric conditions," Journal of Electromyography and Kinesiology, vol. 14, no. 6, pp. 699-707, 2004. 

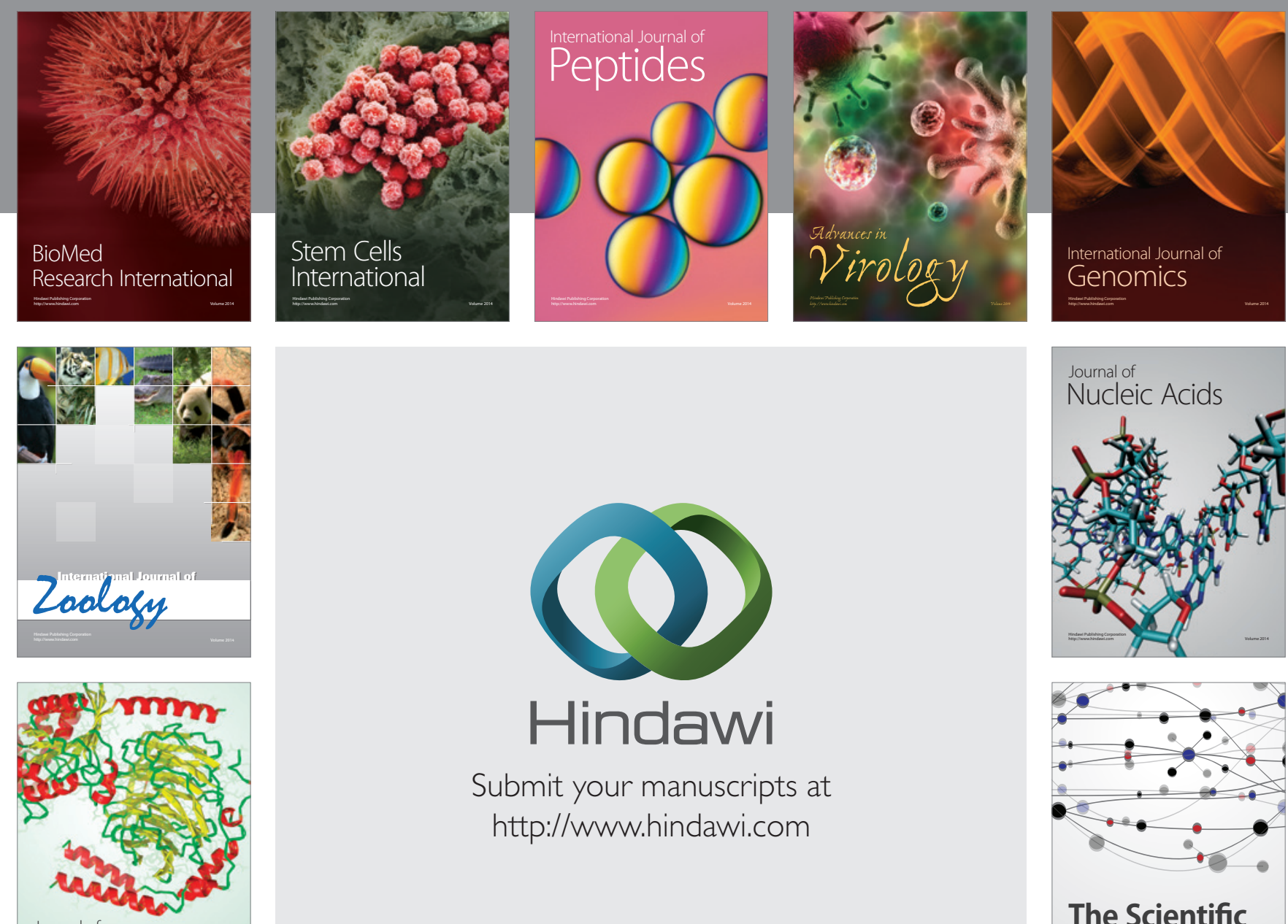

Submit your manuscripts at

http://www.hindawi.com

Journal of
Signal Transduction
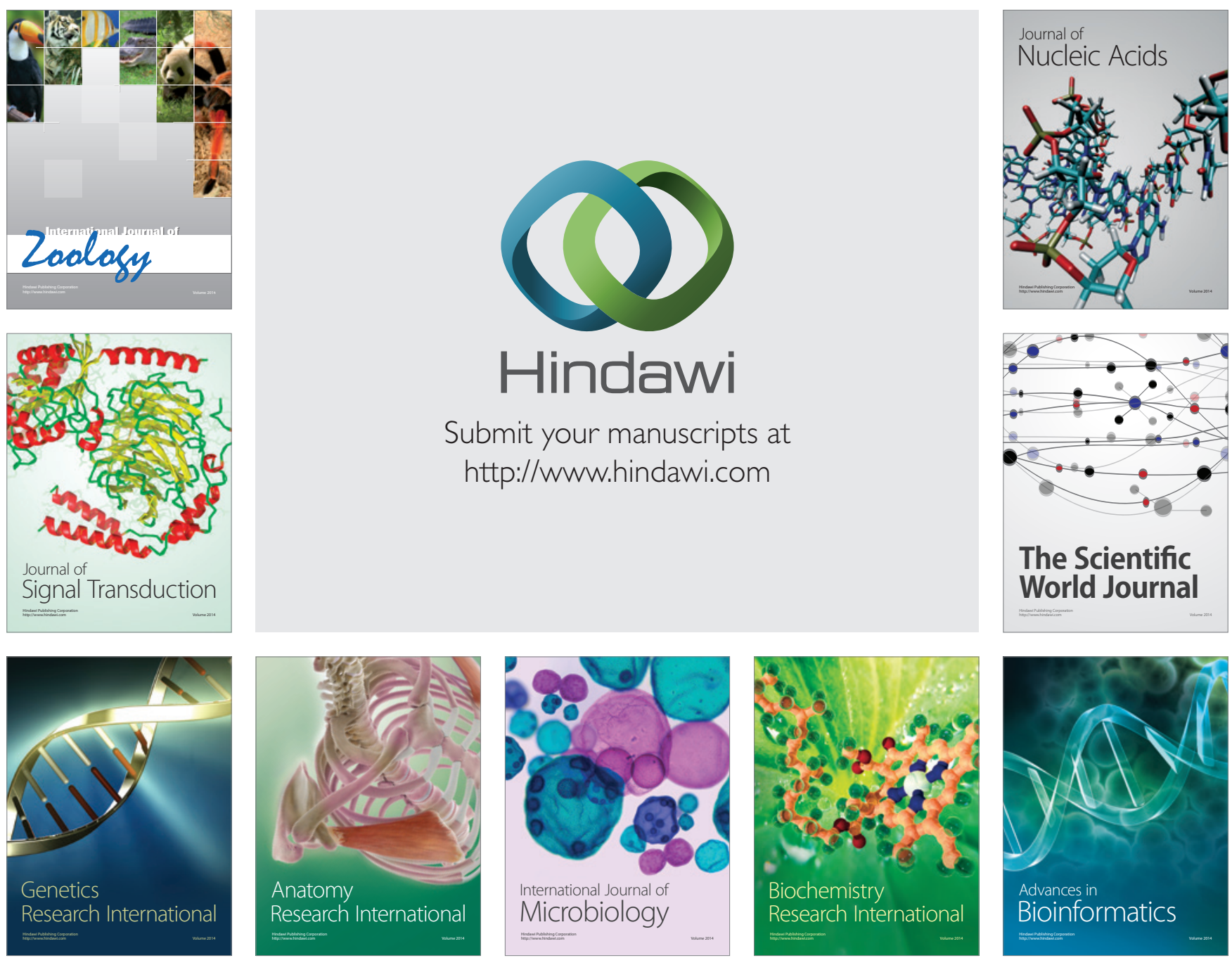

The Scientific World Journal
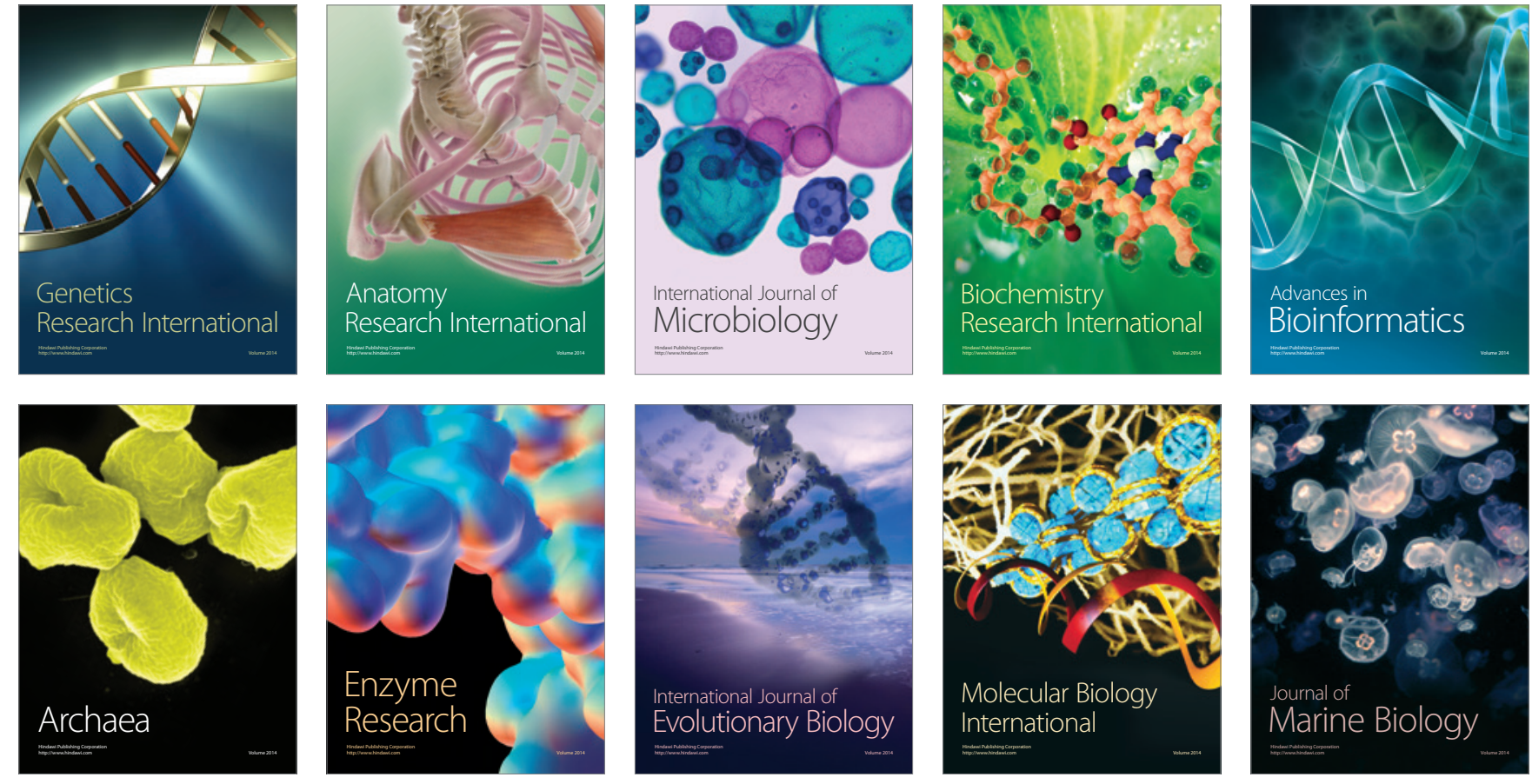